\title{
The pros and cons of the Heart Team
}

\author{
Ran Kornowski*,1,2 \& Guy Witberg ${ }^{1,2}$ \\ ${ }^{1}$ Department of Cardiology, Rabin Medical Center, Petach Tikva, Israel \\ ${ }^{2}$ Faculty of Medicine, Tel Aviv University, Tel Aviv, Israel \\ *Author for correspondence: Tel.: +972 3937 7107; Fax: +972 3924 9850; ran.kornowski@gmail.com
}
"one must recognize that the Heart Team is not a rules-free forum and it should be led according to mutual understanding and shared goals, as decided by its members"

First draft submitted: 5 April 2019; Accepted for publication: 9 April 2019; Published online: 10 July 2019

\section{Keywords: clinical outcomes • coronary revascularization • guidelines • Heart Team • transcatheter aortic valve replacement}

In 2010, for the first time, the Heart Team concept was integrated into the European Society of Cardiology revascularization guidelines [1]. The document was inspired by expert opinion and by the SYNTAX coronary revascularization trial [2]. This trial compared coronary bypass surgery with percutaneous coronary intervention using drug-eluting stents in patients with multivessel and left main coronary artery disease. The enrollment phase of the trial involved shared decision making by interventional cardiologists and cardiac surgeons about eligibility for coronary revascularization procedures [2]. Since that trial, the Heart Team consultation forum has emerged and expanded to various subspecialty disciplines in cardiovascular medicine, including valvular and structural heart diseases. The growing presence of this kind of team is presumably because of its reputation as the best recommended practice tool for determining proper treatment strategies and overall medical care for patients. The most recent edition of the European Society of Cardiology and of the American College of Cardiology coronary revascularization guidelines emphasized the need for a Heart Team, rating this recommendation as Class IC [3,4]. Valve-related guidelines have reached similar conclusions [5]. Although Class IC is the highest recommendation level, it is still somewhat internally inconsistent, as level I indicates the highest possible endorsement, but grade C (i.e., experts' opinion) implies a lack of scientific evidence or rigor to justify the highest ranking.

In this article, we analyze the pros and cons of Heart Team endorsement and implementation, a topic which is not often discussed. For many practitioners, despite the lack of evidence validating such a paradigm, it is quite obvious that if the Heart Team is functional and works properly, the patient should benefit from it. But what if the Heart Team is dysfunctional or does not operate properly? Questions then arise about what could happen to the decision-making process and the professional environment, and if patients outcomes might be compromised.

\section{What makes a functional team?}

The difference between teams that do and do not perform well receives far too little attention from most practitioners. Part of the problem is that 'team' is a word and concept that is familiar and even trivial to everyone, yet in the reality of clinical practice not all professional forums are functional teams. Moreover, teams can be productive or counterproductive. The difference depends on personal or professional issues and the extent of shared goals and good understanding among the team members about the rules of engagement in a medical consultation forum.

The Wisdom of Teams is a book written by Jon R Katzenbach and Douglas K Smith that was published in 1993, then subsequently updated and re-published by Harvard Business Review Press in 2015 [6]. The authors explored this matter extensively by researching factors that differentiate various levels of team performance, where and how teams work best, and what management can do to enhance a team's effectiveness. Their study was based on hundreds of communications with $>50$ different teams in 30 firms, mainly among large companies. Nonetheless, some of their fundamental insights and conclusions can apply in the healthcare sector.

They found that basic discipline makes teams work, and that teams and good performance are inseparable - you cannot have one without the other. However, people use the word 'team' so loosely that the term gets in the way

Future Medicine 
of learning and applying the discipline that leads to good performance. Teamwork represents a set of values that encourage listening and responding constructively to views that other team members express, giving others the benefit of the doubt, providing support, and recognizing the interests and achievements of others. Such values help teams operate and promote individual performance and the performance of an entire organization [6].

Teamwork values by themselves are not enough to ensure team performance, however. Members of the team must also share similar goals. All team members need to recognize their mutual urgent and worthwhile purpose, mission, acknowledge their responsibilities and what the expectations are. They also need to trust and respect each other, use their skills and expertise in a complementary and synergistic way, put aside professional egos, be ready to act together and even compromise when needed to achieve optimal outcomes. The team authority should be defined as the rule of conduct on the most critical issues, the decision-making process and the execution of these decisions. Effective teams trace the progress and track the execution and outcomes of their decisions. The ultimate goal of teams is to use the collective expertise and work product to deliver superlative performance. Ideally, the working group performance should be greater than the cumulative potential of its individual members. Such collective work product reflects the joint, real contribution of the team members.

\section{Applying it to the Heart Team}

Now let's get back to the Heart Team forum and think through the process and dialog that exist between cardiologists and cardiac surgeons or other members of the professional team. Without doubt, all involved parties are aiming for the best possible outcomes for their patients; this is an axiom. In some instances, team members find it relatively easy to discuss a clinical scenario and obtain good insight into next steps, making a joint decision about the procedural strategy (e.g., coronary artery bypass graft versus percutaneous coronary intervention versus optimal medical treatment only, or surgical versus transcatheter aortic valve replacement [TAVR] or deferred any intervention). In this case, a shared decision by the Heart Team, acting in consensus, is probably the best possible professional and clinical scenario.

But what happens without such a straightforward case? The process might be more fraught if the interpersonal relationships within the Heart Team are rather 'toxic', with professional conflicts or personal motivations in play. Income incentives, a solo stance (e.g., 'I know best'), or merely different interpretation of scientific data or clinical guidelines could create obstacles. The result might be disagreement among members of the consultation forum about the best recommended therapy for patients. Such a case could be problematic for the Heart Team but more so for the patient. In this negative scenario, the question arises of whether the Heart Team is truly beneficial or if there is an alternative approach for achieving the best medical practice and outcome.

Additional shortcomings should be considered before Heart Team decisions become the arbiter of care. The most troubling is the lack of reproducibility concerning data interpretation and decision-making processes among such teams. Team members reflect and characterize the team, and one group might differ from another in terms of interpretation of the clinical situation, thus reaching a different recommendation for the patient. In addition, diverse teams may differ in their professional attitude or level of expertise, so that their output could reflect local know-how rather than an 'ideal' medical setting. To the best of our knowledge, the consistency and reproducibility of Heart Team decisions and recommendations have been formally tested in a single small study from the UK [7]. The reproducibility data were based on re-discussion of 40 cases by the Heart Team 9 months post the initial discussion; in $80 \%$ of cases the outcome of the consultation was identical to the initial recommendation but in $20 \%$ the team gave a different recommendation upon exactly the same patient/case. Interestingly, the same team also found that in $93 \%$ of cases discussed, their decisions were actually implemented in practice. In theory, the same patient could receive a specific recommendation from one team but a different endorsement from another, despite having the same medical problem.

\section{Assessing the Heart Team}

The question at hand is what metrics can be used to identify a 'functional' from a 'dysfunctional' Heart Team? The goal is to avoid inconsistencies within groups and between groups. We propose herein that the Heart Team should have a periodic assessment of three key statistics: applicability, internal validity and external validity. The internal validity could be derived from re-examining past cases added to the regular Heart Team discussions without the participants knowing that these cases were actually already discussed and treated some time (e.g., $>6$ months) ago. In addition, a periodical assessment by a second Heart Team (from a similar grade center) might be advisable to attain data on the external validity of the Heart Team decisions. One further step that can be taken to gauge 
whether there is a problem within the institutional decision-making process is to compare the clinical outcomes of patients discussed to national reference standards, this is harder to do and of course mandates that national databases of outcomes are available, which is not always the case. At the moment we cannot define any thresholds for reproducibility and applicability of Heart Team decision, but it is obvious that for quality assurance such a process needs to be applied and $80 \%$ might be a reasonable empirical threshold. If for example, a Heart Team finds that only $50 \%$ of its decisions are eventually applied or that the internal validity (reproducibility) of its recommendations is just 50\% - this should raise a serious 'red flag' and lead to a process of self assessment in an attempt to identify the causes for these poor results. We assume that in many institutions the enthusiasm to invest the time and resources to hold such an audit process will not be prodigious, but the desired and optimal solution should be considered before strongly endorsing the Heart Team applicability to current practice.

\section{Who makes the ultimate decision?}

The Heart Team might also look like a challenge to the competence of cardiologists to undertake independent decisions about therapeutic strategies, jeopardizing their ability to impact patients' outcomes. In many instances, the cardiologists have long-term relationships with patients because of the chronic nature of most cardiac diseases. Now, in a pivotal moment or clinical exacerbation, turning to a Heart Team could suggest that experienced cardiologists, who are trained to handle such a crisis, cannot make a firm independent decision about optimal patient management. The decision could ultimately be to refer the patient for surgical intervention or involving the surgeon for a Heart Team discussion. The question is whether it is necessary to give the Heart Team the ultimate mandate to undertake such a decision. The immediate counter argument might be that the fact that a cardiologist has a long standing relationship with a patient also has a potential biased effect and that a 'fresh' set of professional eyes, using the multidisciplinary Heart Team approach can give a more impartial and optimized assessment of the case.

Moreover, it is unclear what medical centers without cardiac surgical departments are to do if Heart Team consultation is indeed mandatory. How could these cardiology practices operate best for the benefit of their patients, and what communication tools are needed to implement the recommended guidelines in those institutions? The latter point is more of a technical telecommunication issue as according to our knowledge, in many parts of the world, district hospitals are discussing cases with Heart Teams from tertiary referral centers regarding the optimal therapeutic strategy and it seems to be working quite well.

The Heart Team consultation could and should work both ways. The team should bring to the table both interventional and surgical candidates because over time, paradigms can shift regarding mode of treatment of various cardiovascular diseases. Taking TAVR as an example, two studies recently showed that low-risk patients could benefit from catheter-based interventions rather than having open heart aortic valve replacement surgery [8,9]. Thus, it seems that soon, Heart Teams would need to discuss low-risk aortic stenosis patients who traditionally have undergone open-heart surgery but now could potentially benefit from TAVR. Similar considerations should be applied for patients with functional mitral insufficiency, based on results of COAPT trial [10], and for other structural interventions.

\section{Successfully utilizing the Heart Team}

The most important issue is how to best use the Heart Team asset for the benefit of patients and how to form a synergistic professional environment geared exclusively toward identifying the best therapeutics. Even without proof of concept, it seems reasonable and logical to assume a great advantage from having a competent Heart Team in your hospital, working constructively and collegially so that patients could certainly benefit. Nonetheless, one must recognize that the Heart Team is not a rules-free forum and it should be led according to mutual understanding and shared goals, as decided by its members. As highlighted above, quality assurance metrics should be applied to the workflow of the Heart Team. It is probably better not to have a Heart Team at all if it is dysfunctional or confrontational, and in such cases alternative pathways should be sought to maintain optimized medical care and/or improve the Heart Team operation itself. Heart Teams must establish their codes of conduct and practices, using the tools and skills that they will need after they are formed to optimize team performance. Successful teams set performance standards and survey the outcomes for their patients according to their recommendations. The Heart Team is an extremely important forum that should maintain its mission of responsibility and remain highly professional for the benefit of patients. 
Financial \& competing interests disclosure

The authors have no relevant affiliations or financial involvement with any organization or entity with a financial interest in or financial conflict with the subject matter or materials discussed in the manuscript. This includes employment, consultancies, honoraria, stock ownership or options, expert testimony, grants or patents received or pending, or royalties.

No writing assistance was utilized in the production of this manuscript.

\section{References}

1. Wijns W, Kolh P, Danchin N et al. Guidelines on myocardial revascularization. Eur. Heart J. 31(20), 2501-2555 (2010).

2. Serruys PW, Morice MC, Kappetein AP et al. Percutaneous coronary intervention versus coronary-artery bypass grafting for severe coronary artery disease. N. Engl. J. Med. 360(10), 961-972 (2009).

3. Neumann FJ, Sousa-Uva M, Ahlsson A et al. 2018 ESC/EACTS guidelines on myocardial revascularization. Eur. Heart J. 40(2), 87-165 (2019).

4. Levine GN, Bates ER, Blankenship JC et al. 2011 ACCF/AHA/SCAI guideline for percutaneous coronary intervention. A report of the American College of Cardiology Foundation/American Heart Association Task Force on Practice Guidelines and the Society for Cardiovascular Angiography and Interventions. J. Am. Coll. Cardiol. 58(24), e44-e122 (2011).

5. Baumgartner H, Falk V, Bax JJ et al. 2017 ESC/EACTS Guidelines for the management of valvular heart disease. Eur. Heart J. 38(36), 2739-2791 (2017).

6. Katzenbach JR, Smith DK. The Wisdom of Teams: Creating the High-Performance Organization. Harvard Business Review Press (2015).

7. Pavlidis AN, Perera D, Karamasis GV et al. Implementation and consistency of Heart Team decision-making in complex coronary revascularisation. Int. J. Cardiol. 206, 37-41 (2016).

8. Mack MJ, Leon MB, Thourani VH et al. Transcatheter aortic-valve replacement with a balloon-expandable valve in low-risk patients. $N$. Engl. J. Med. 380(18), 1695-1705 (2019).

9. Popma JJ, Deeb GM, Yakubov SJ et al. Transcatheter aortic-valve replacement with a self-expanding valve in low-risk patients. $N$. Engl. J. Med. 380(18), 1706-1715 (2019).

10. Stone GW, Lindenfeld J, Abraham WT et al. Transcatheter mitral-valve repair in patients with heart failure. N. Engl. J. Med. 379(24), 2307-2318 (2018). 